TOPIC FOR DISCUSSION

\title{
What is the evidence behind the evidence-base? The premature death of block-replace antithyroid drug regimens for Graves' disease
}

\author{
Salman Razvi ${ }^{1}$, Bijay Vaidya ${ }^{2}$, Petros Perros ${ }^{3}$ and Simon H S Pearce ${ }^{1,4}$ \\ ${ }^{1}$ Department of Endocrinology, Royal Victoria Infirmary, Newcastle upon Tyne, UK, ${ }^{2}$ Department of Endocrinology and Diabetes, Royal Devon and Exeter \\ Hospital, Exeter, UK, ${ }^{3}$ Department of Endocrinology, Freeman Hospital, Newcastle upon Tyne, UK and ${ }^{4}$ Institute of Human Genetics $\&$ School of Clinical \\ Medical Sciences, University of Newcastle, Centre for Life, Central Parkway, Newcastle upon Tyne, NE1 3BZ, UK
}

(Correspondence should be addressed to S Pearce at the Institute of Human Genetics, University of Newcastle, UK; Email: s.h.s.pearce@ncl.ac.uk)

\begin{abstract}
Block-replace and titration antithyroid drug regimens both give similar rates of medium- to long-term remission of hyperthyroid Graves' disease. Recent meta-analysis, however, has suggested that titration regimens may be preferable owing to a higher rate of adverse events seen in the block-replace arms of published comparative studies. This article critically re-evaluates the evidence upon which these metaanalyses were based. We suggest that there is little objective evidence that is pertinent to current clinical practice to separate block-replace from titration antithyroid drug regimens and that both remain satisfactory approaches to the medical management of hyperthyroid Graves' disease.
\end{abstract}

European Journal of Endocrinology 154 783-786

\section{Background}

It is imperative that physicians look carefully at the evidence that guides their clinical practice. This evidence may be derived from many sources, ranging from a rigorous, randomised controlled trial (RCT), through to the reported experiences of respected colleagues. Individual RCTs are rarely identical in terms of protocol, characteristics of enrolled patient groups or clinical outcomes. So where several RCTs have been performed, meta-analysis has also become a common means to assess the average benefit or hazard that a practitioner might expect from using a treatment. Such meta-analysis should represent the best possible summation of the evidence and may be appropriately endorsed by publication in an authoritative journal or by being included in a peer-reviewed collection of evidence-based analysis such as the Cochrane Library Collection. These works are important benchmarks for good practice and in a world where there is increasing external regulation of medical care, such meta-analyses are taken as gold-standard evidence by those commissioning healthcare and thus may even influence the range of treatments that are available in managed care settings. They may also form the basis for legal representations following an adverse outcome of care. Thus, it is critical that meta-analyses are conducted rigorously, and that their results are interpreted in a fashion appropriate to the evidence base (1). In this paper we comment on the interpretation of the widely published meta-analyses of trials of antithyroid drug treatment for Graves' disease $(2,3)$. A key conclusion of these analyses is that titrated antithyroid drug regimens are preferable to blockreplace treatment protocols, and it is this conclusion that we challenge.

\section{Analysis}

In the UK, it is common practice to initiate drug therapy of hyperthyroid Graves' disease with $40 \mathrm{mg}$ daily of carbimazole. In countries where methimazole is available, $30 \mathrm{mg}$ is the frequent daily starting dose (4). Although Abrahams and colleagues comment that methimazole and carbimazole have 'dose equivalence', in fact the compounds have molar dose equivalence. However, as carbimazole has a higher molecular mass than methimazole (186 vs 114) the administered doses are not equivalent, with $6.1 \mathrm{mg}$ methimazole having the same in vivo effect as about $10 \mathrm{mg}$ carbimazole (5). After 6-8 weeks of treatment with methimazole $30 \mathrm{mg}$ daily or carbimazole $40 \mathrm{mg}$ daily, the majority of subjects with hyperthyroid Graves' disease have become euthyroid (as judged by normal serum-free thyroid hormone concentrations). At this stage treatment can be continued either with a block-replace or a titrated dose antithyroid drug regimen. For a block-replace regimen, a replacement dose of thyroxine is added, and the antithyroid drug is continued in an unchanged dose. In a titrated dose regimen, the thionamide dose is 
reduced, and the endogenous production of thyroxine is maintained from the partial block to thyroid hormone synthesis. It is clear that there is little to choose between titrated and block-replace antithyroid drug regimens in terms of long-term outcome of Graves' disease. Twenty-four months after stopping treatment, both methods leave approximately $50 \%$ of subjects in medium- to long-term remission $(2,3,6-8)$. Thus, the evidence cited by Abrahams and colleagues to favour titrated antithyroid drug regimens over block-replace regimens comes exclusively from the higher rate of adverse events found in the block-replace arms of the trials examined by the meta-analysis. We would like to comment on several features of this analysis, focusing initially on these adverse events.

Some of the adverse effects of thionamide drug treatment may be regarded as minor, such as rash or episodes of hypothyroidism on treatment, which are inconvenient for the patient (9). Nonetheless, these minor adverse events may lead to withdrawal of drug therapy, and hence result in a failure of medical treatment. However, it is the serious adverse event of agranulocytosis that is of primary concern, and there is already good evidence that the prevalence of this feared complication is dependent upon the dose of antithyroid drug $(10,11)$. For instance, Wiberg and Nuttall's study of 25 patients with hyperthyroidism treated with methimazole $120 \mathrm{mg}$ daily found that three $(12 \%)$ developed neutropenic fever or agranulocytosis (10). Similarly, in the randomised study of Grebe et al., which is included in the meta-analysis, 2 of 17 Graves' patients (12\%) treated with carbimazole $100 \mathrm{mg}$ daily for 6 months also developed agranulocytosis (11). These high rates of neutrophil dyscrasia observed with high-dose thionamide treatment need to be compared with a prevalence of about $0.4 \%$ from a prospectively monitored patient cohort on conventional antithyroid drug doses (12). In the meta-analysis, seven studies were used to provide evidence of the adverse effects of the antithyroid drug regimens (the publications of Reinwein and Benker report the same clinical cohort) $(8,11,13-18)$. However, six of these seven had used thionamide doses in the block-replace arm that are significantly higher than would now represent conventional clinical practice (Table 1). Indeed, the stated aim of several of these studies was to determine whether improved remission rates could be obtained from higher dose treatment, not to compare the tolerability of the two regimens $(11,14)$. Thus only one out of the seven studies analysed are relevant to current clinical practice in employing a conventional starting dose of thionamide for a block-replace regimen (16). While it is undoubtedly true that collectively these studies showed a higher rate of adverse events in the block-replace arms, it is also clear that these studies are not representative of current mainstream clinical practice, as only in exceptional circumstances (e.g. cardiac compromise, thyrotoxic storm) would most patients be started on more than $40 \mathrm{mg}$ carbimazole or equivalent. Therefore, we feel that the conclusion that a titrated antithyroid drug regimen should be considered the optimal first-line drug treatment for Graves' disease cannot be supported on this basis.

In an attempt to analyse this issue further, we reexamined the original publications and reconsidered some of the analyses. We were surprised to find that the data extracted from one of the original publications had been misrepresented. In the study of Edmonds \& Tellez (13), all 70 Graves' disease patients were treated for an initial period of 4 weeks with carbimazole $60 \mathrm{mg}$ daily, and then the treatment arms diverged to block-replace or titration regimens. Nine patients with rash were reported during the initial 4 week period, without details of their treatment allocation in the original report. During the rest of the study, two patients on block-replace and one on titrated dose carbimazole were reported to have developed rash (12 episodes of rash in total). In the meta-analysis $(2,3)$, only nine episodes of rash are included and all are categorised as having occurred on block-replace producing the most extreme deviation from statistical normality of all the studies. Repeating the meta-analysis using the amended data on rash from this study (seven episodes on block-replace vs five with titration; C J Edmonds, personal communication) subtly alters the conclusions. Indeed, if these revised data are included, and we exclude the two studies that are clearly 'outliers' to current practice in terms of the dose of thionamide used (those using carbimazole $100 \mathrm{mg}$ or methimazole $60 \mathrm{mg}$ daily $(11,14))$, there is no significant difference in the rate

Table 1 Studies comparing the two treatment regimens of antithyroid medication and their doses.

\begin{tabular}{|c|c|c|c|}
\hline Author; year & $\begin{array}{c}\text { Drug dose in block-replace arm } \\
\text { (mg/day) }\end{array}$ & $\begin{array}{l}\text { Carbimazole dose equivalent } \\
\qquad(\mathrm{mg} / \text { day })^{\star}\end{array}$ & Reference \\
\hline Edmonds \& Tellez; 1994 & Carbimazole 60 & - & 13 \\
\hline Leclere; 1994 & Carbimazole 60 & - & 15 \\
\hline Jorde et al.; 1995 & Methimazole 60 & 98 & 14 \\
\hline Wilson et al.; 1996 & Carbimazole 60 & - & 17 \\
\hline Grebe et al.; 1998 & Carbimazole 100 & - & 11 \\
\hline Benker et al.; 1998 & Methimazole 40 & 66 & 8 \\
\hline Nedrebo et al.; 2002 & Carbimazole 29† & - & 16 \\
\hline
\end{tabular}

*Based on dose equivalence reported by Jansen et al. (5). †Mean daily dose. 


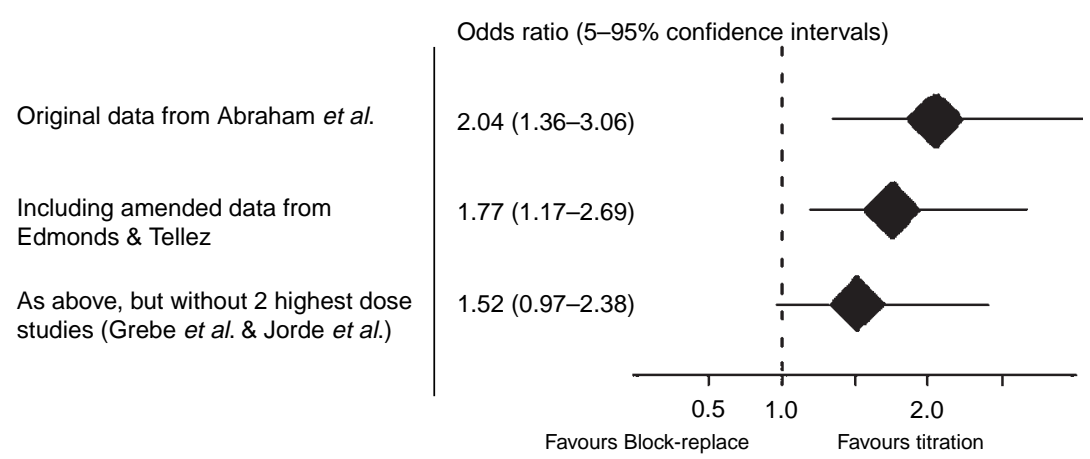

Figure 1 Meta-analysis showing prevalence of rash during antithyroid drug trials. Odds ratios $\pm 5-95 \%$ confidence intervals for the prevalence of rash during antithyroid drug trials. The top line recapitulates the data from the seven trials examined in the meta-analyses by Abraham et al. $(2,3)$. The second line shows data from the same seven studies but includes the amended data from the Edmonds \& Tellez study (13). The bottom line shows data on rash from the five studies that used lower doses of antithyroid drug (Leclere, Edmonds \& Tellez, and Wilson et al. used carbimazole $60 \mathrm{mg}$ daily; Benker et al. used methimazole $40 \mathrm{mg}$ daily and Nedrebo et al. used carbimazole in a mean dose of $29 \mathrm{mg}$ daily) $(8,13,15-17)$. As can be seen, with the amended data and the very high dose trials excluded, there is no significant difference in the prevalence of rash.

of rash between the two groups (odds ratio 1.52, 5-95\% confidence intervals 0.97-2.38) (Fig. 1). This suggests that the benefit of the titration regimen over block-replace for the adverse event of rash may be marginal at currently employed doses.

Concerning the important adverse event of agranulocytosis, there were 12 episodes reported from five studies, encompassing a total of 943 patients. Of these 12 episodes, four (three block-replace, one titrated) were reported in the 93 patients in the two highest dose studies; those using carbimazole $100 \mathrm{mg}$ or methimazole $60 \mathrm{mg}$ daily $(11,14)$. Overall, there was no significant difference in the rate between either regimen; however, it needs to be borne in mind that these studies were not of sufficient power to detect a real difference in agranulocytosis rate (around 30\% collective power to detect a true doubling of agranulocytosis events at $\alpha=0.05$, given the numbers of patients). Furthermore, the peak time for agranulocytosis is during the 4th to 6th week of antithyroid drug therapy (19), at a time when the majority of subjects will be on the same dose of antithyroid drug, whichever regimen the physician intends to use.

Thus, under close scrutiny, the evidence to favour titration antithyroid regimens over block-replace owing to differences in adverse event rate seems unconvincing, particularly so given the more modest doses of these compounds currently employed in block-replace regimens. The dose of antithyroid drug used in the studies that were meta-analysed is not representative of everyday practice. Other factors that were not directly addressed in the meta-analysis may also be relevant. First, prolonged periods of mild to moderate hypothyroidism can be considered a significant adverse event related to titrated antithyroid drug regimens, which rarely occurs during block-replace treatment. The Cochrane review (2) acknowledges that no trial provides any data about this predictable side-effect, which may be an important factor in the progression of thyroid eye disease (20) and possibly for future vascular risk (21). Furthermore, hypothyroidism may contribute to significant weight gain, which is subsequently difficult to reverse. In addition, about $20 \%$ of subjects with Graves' hyperthyroidism may have antibodies directed against the thyroid-stimulating hormone (TSH) receptor that have blocking effects, as well as the usual stimulatory antibodies. In these subjects, spontaneous fluctuations in the degree of antibodyinduced thyroid stimulation may be difficult to control with a titrated regimen, whereas such fluctuations are rarely observed in subjects on block-replace regimens. Although there are no studies comparing cost effectiveness, it is our impression that patients on a blockreplace regimen require less frequent clinical and biochemical monitoring of thyroid function than a titrated dose regimen. In the UK, the cost to the NHS for a year of titration dose regimen with carbimazole (40 mg for 6 weeks, followed by $20 \mathrm{mg}$ for 8 weeks, $10 \mathrm{mg}$ for 12 weeks and eventually $5 \mathrm{mg}$ for 26 weeks) is $£ 28.70$ compared with $£ 105.85$ for the same duration of block-replacement $40 \mathrm{mg}$ carbimazole and $100 \mu \mathrm{g}$ thyroxine (22). It is likely that the latter regimen will be cheaper overall, since additional clinic visits and biochemical testing will more than offset the extra drug costs.

\section{Conclusion}

In summary, we suggest that block-replace antithyroid drug regimens, using modest daily doses of antithyroid agents (i.e. carbimazole $40 \mathrm{mg}$ daily or equivalent) remain an excellent approach to managing Graves' disease. For patients with thyroid eye disease, or those at risk of this complication, for those with fluctuant thyroid function during treatment and for those in 
whom frequent blood testing is unavailable or undesirable, we feel that block-replace regimens may have significant advantages as medical therapy for hyperthyroid Graves' disease. We support the other recommendations of the meta-analysis, in particular that radioiodine therapy should be discussed at an early stage with subjects who have moderate to severe hyperthyroidism without thyroid eye disease. We also agree that adequately powered trials of titration and block-replace regimens, with commonly used doses of antithyroid drug, would provide a better evidence base for decision making.

\section{Acknowledgements}

We are grateful to Dr C J Edmonds for sharing data with us. We understand that an amendment to the Cochrane review (2) will be undertaken at the next online revision (P Abraham, personal communication).

\section{References}

1 Leandro G. Meta-analysis in Medical Research: The Handbook for the Understanding and Practice of Meta-analysis Oxford: Blackwell Publishing, 2005.

2 Abraham P, Avenell A, Watson WA, Park CM \& Bevan JS. Antithyroid drug regimen for treating Graves' hyperthyroidism. Cochrane Database Systematic Review 2005 (2): CD003420

3 Abraham P, Avenell A, Park CM, Watson WA \& Bevan JS. A systematic review of drug therapy for Graves' hyperthyroidism. European Journal of Endocrinology 2005153 489-498.

4 Cooper DS. Antithyroid drugs. New England Journal of Medicine 2005352 905-917.

5 Jansson R, Dahlberg PA \& Lindstrom B. Comparative bioavailability of carbimazole and methimazole. International Journal of Clinical Pharmacology and Therapeutic Toxicology 198321 505-510.

6 Weetman AP, Ratanachaiyavong S, Middleton GW, Love W, John R, Owen GM, Darke C, Lazarus JH, Hall R \& McGregor AM. Prediction of outcome in Graves' disease after carbimazole treatment. Quarterly Journal of Medicine 198659 409-419.

7 Solomon DH, Beck JC \& Vanderlaan WP. Prognosis of hyperthyroidism treated by antithyroid drugs. Journal of the American Medical Association 1953152 201-205.

8 Benker G, Reinwein D, Kahaly G, Tegler L, Alexander WD, Fassbinder $\mathrm{J} \&$ Hirche $\mathrm{H}$. Is there a methimazole dose effect on remission rate in Graves' disease?, Results from a long-term prospective study. The European Multicentre Trial Group of the Treatment of Hyperthyroidism with Antithyroid Drugs. Clinical Endocrinology $1998 \mathbf{4 9} 451-457$.
9 Bartalena L, Bogazzi F \& Martino E. Adverse effects of thyroid hormone preparations and antithyroid drugs. Drug Safety 1996 15 53-63.

10 Wiberg JJ \& Nuttall FQ. Methimazole toxicity from high doses. Annals of Internal Medicine 197277 414-416.

11 Grebe SK, Feek CM, Ford HC, Fagerstrom JN, Cordwell DP, Delahunt JW \& Toomath RJ. A randomized trial of short-term treatment of Graves' disease with high-dose carbimazole plus thyroxine versus low-dose carbimazole. Clinical Endocrinology $199848585-592$.

12 Tajiri J, Noguchi S, Murakami T \& Murakami N. Antithyroid druginduced agranulocytosis, The usefulness of routine white blood cell count monitoring. Archives of Internal Medicine 1990150 621-624.

13 Edmonds CJ \& Tellez M. Treatment of Graves' disease by carbimazole: high dose with thyroxine compared to titration dose. European Journal of Endocrinology 1994131 120-124.

14 Jorde R, Ytre-Arne K, Stormer J \& Sundsfjord J. Short-term treatment of Graves' disease with methimazole in high versus low doses. Journal of Internal Medicine 1995238 161-165.

15 Leclere J. Treatment of Basedow disease with synthetic antithyroid drugs. Evaluation of the dose on the efficacy of the long term treatment. Annales d'Endocrinologie 199455 11-14.

16 Nedrebo BG, Holm PI, Uhlving S, Sorheim JI, Skeie S, Eide GE, Husebye ES, Lien EA \& Aanderud S. Predictors of outcome and comparison of different drug regimens for the prevention of relapse in patients with Graves' disease. European Journal of Endocrinology $2002147583-589$.

17 Wilson R, Buchanan L, Fraser WD, McKillop JH \& Thomson JA. Do higher doses of carbimazole improve remission in Graves' disease? Quarterly Journal of Medicine $199689381-385$.

18 Reinwein D, Benker G, Lazarus JH \& Alexander WD. A prospective randomized trial of antithyroid drug dose in Graves' disease therapy. European Multicenter Study Group on Antithyroid Drug Treatment. Journal of Clinical Endocrinology and Metabolism 1993 76 1516-1521.

19 Pearce SH. Spontaneous reporting of adverse reactions to carbimazole and propylthiouracil in the UK. Clinical Endocrinology $200461589-594$.

20 Tallstedt L, Lundell G, Blomgren H \& Bring J. Does early administration of thyroxine reduce the development of Graves' ophthalmopathy after radioiodine treatment? European Journal of Endocrinology 1994130 494-497.

21 Franklyn JA, Sheppard MC \& Maisonneuve P. Thyroid function and mortality in patients treated for hyperthyroidism. Journal of the American Medical Association 2005294 71-80.

22 Health and Social Care Information Centre. Prescription cost analysis: England, 2004. Department of Health publication. http://www.dh.gov.uk/assetRoot/04/10/76/26/04107626.pdf Accessed 15 March 2006.

Received 1 March 2006

Accepted 21 March 2006 